\title{
SensorFINT, the new European Network for assuring food integrity using non-destructive spectral sensors

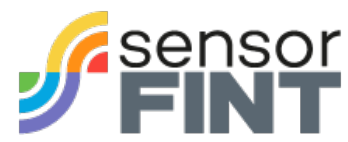

\section{Lola Pérez-Marín}

Chair of SensorFINT COST Action, Faculty of Agriculture and Forestry Engineering (ETSIAM), University of Córdoba, Campus Rabanales, 14071 Córdoba, Spain

The SensorFINT COST Action (CA 19145) is a European Network for assuring food integrity using non-destructive spectral sensors. SensorFINT is funded by the European Cooperation in Science and Technology (COST) organisation with the primary objective of promoting the creation of research networks in innovative areas, facilitating the collaboration between academia and industry in Europe and beyond.

Our main objective is to create a vibrant and multidisciplinary network focused on the application of non-destructive spectral sensors to solve the demands of industry for assessment of the quality, safety, authenticity and traceability of food, combining experience in research, manufacture, training and technology transfer to accelerate spectral sensor implementation throughout the food chain. Thus, the Action is oriented to the use of sensors and technologies that allow the design of new, intelligent quality control systems to achieve the new challenges of Industry 4.0. That is, a widespread and instantaneous non-destructive inspection of food products to enable decisions to be taken in real time from "field to fork", providing

\section{DOI: $10.1255 /$ sew.2021.a 13}

(c) 2021 The Author

Published under a Creative Commons BY licence

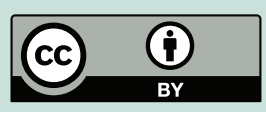

a unique digital fingerprint of the product and, therefore, a new digital labelling strategy with a more complete information about the product for consumers.

Currently, there is an increasing need for the food industry to provide information on their products in order to satisfy consumer demands, quality standards, to produce more and to protect their products from food fraud. Recent developments in technology, and advances in big data analytics, provide the opportunity for step-changes that can transform the role of food integrity assurance from one of just strictly conformance to one that addresses a wide range of business-critical concerns, including quality, safety and authenticity solutions. Non-Destructive Spectroscopic Sensors (NDSS), such as near infrared (NIR) spectroscopy, fluorescence, Raman or hyperspectral imaging, enable rapid, non-destructive and environmentallysafe assessment of multiple parameters in a variety of food products. Most applications of these technologies in the food industry are carried out in the laboratory. Nevertheless, industry requires them to be deployed in situ, i.e. on-line, in-line, on-farm, in-field, for full process control over the entire food chain. These requirements introduce constraints on sensor design and calibration development, which do not normally apply to laboratory-based instruments. Long-term stability of instruments, robustness of calibrations, sensor integration in production environments, transferability of data and the building of real-time decisionmaking systems are critical issues to be considered.

In this Action, specific consideration has been given to those topics, challenges or key issues in NDSS for research and innovation that are still unaddressed or, at least, only partially addressed, such as:

- The integration of several spectral sensors (NIR, fluorescence, Raman etc.), especially combined with imaging systems, to solve critical issues in the agro-food sector. Therefore, the Action tackles the scientific breakthroughs necessary for the fusion of NDSS so that they can be implemented in many parts of the food chain, regardless of the demonstrated development of each technology separately for different food applications.

- The role of NDSS for the widespread sampling of bulk products and batches, highlighting one of their main advantages, which has not yet been fully exploited, the possibility of reducing the sampling error and, therefore, the total analytical error, providing a more precise answer.

- The development of ready-to-use analytical systems, based on the integration and combination of lowcost, portable and miniature NDSS, and information and communication technologies (ICT) for process control and voluntary labelling, to guarantee the integrity and reputation of high added-value products. 


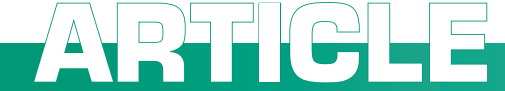
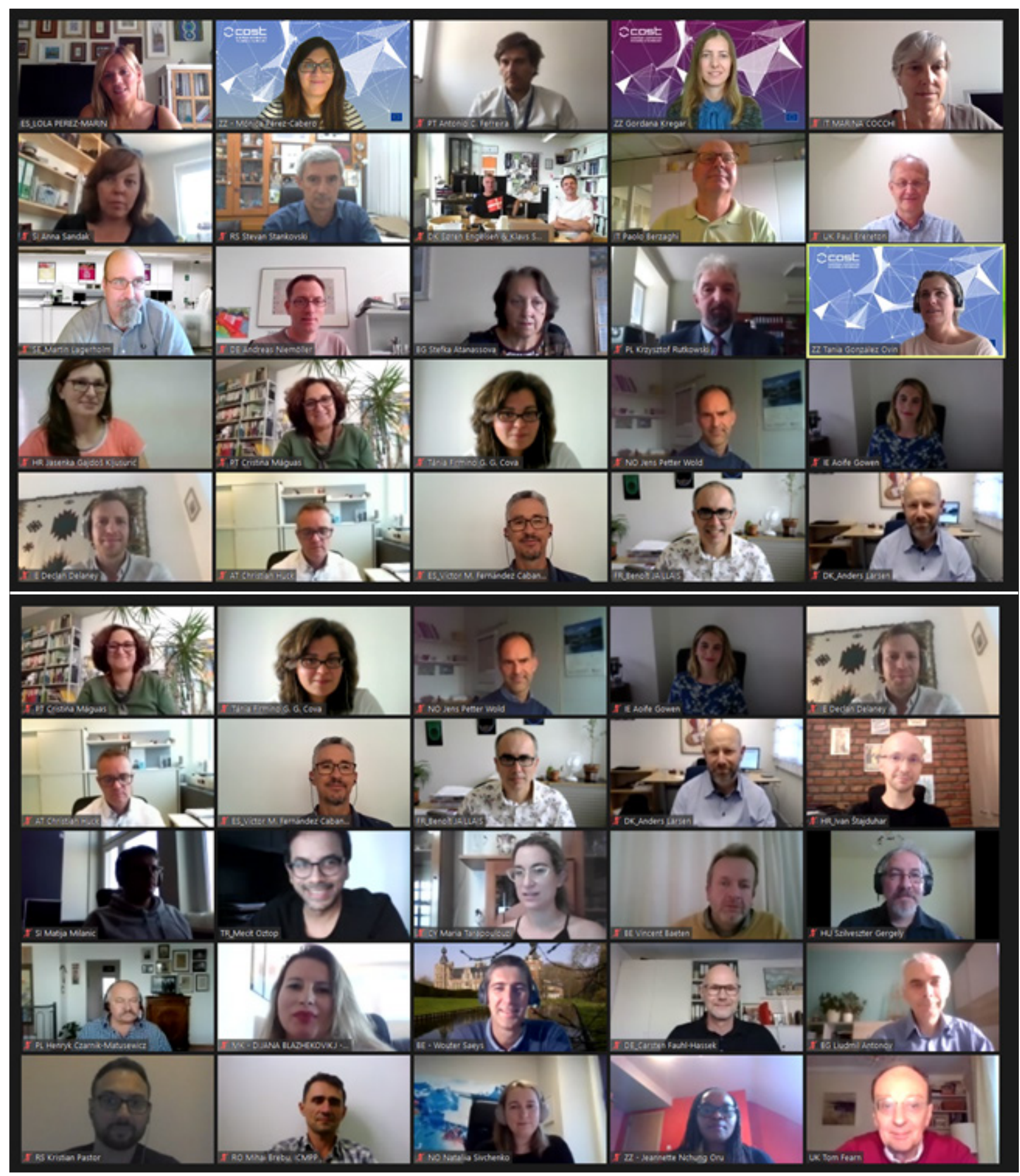

Figure 1. Kick-off meeting of SensorFINT via Zoom.

- The potential of NDSS as non-targeted methods for the individual control of a product item, providing a product fingerprint as a valuable tool for food authentication and fraud prevention.
The SensorFINT Action will manage these NDSS challenges for the demands of the food industry, advancing the stateof-the-art in the technologies and contributing to advances in NDSS applications.
This will involve different strengths and expertise, turning innovative ideas and breakthroughs into new products, solutions and applications. The project is structured into five Working Groups: 


\section{Afintighe-}

1) NDSS for innovation in process control and labelling in the European food industry.

2) Innovation related to the integration of multiple NDSS methods for critical issues in food integrity.

3) Novel mathematical algorithms and methods for processing NDSS in real time.

4) Use of ICT in building decision support systems for the industrial implementation of NDSS.

5) Dissemination and exploitation.

Under the framework of SensorFINT,

a range of activities (conferences, workshops, training schools, exchanges and other dissemination activities) will be organised and funded to promote the culture of NDSS, their implementation in industry and to train future researchers and specialists in the field.

The SensorFINT Action is an open network, now involving more than 30 countries and 150 researchers from Europe and beyond, and it still welcomes new researchers working on spectral sensors, data processing or ICT. Networking activities of the Action, without any doubt, will promote the exchange of information between European and International partners, the dissemination of results and the training of young researchers, who will convert scientific results into a reality that matches industrial needs.

The Action started officially at the beginning of October 2020 (Figure 1) and will continue for four years. More information on all the events being organised, the members of the Action, the travel grants (STSM, Short-Term Scientific Missions) and much more could be found on our website, www. sensorfint.eu (Figure 2). Also, you may follow us on social media (Twitter, Instagram, Linkedin or Facebook) and be updated daily with all the information about SensorFINT's activity.

My last message, please book for our First International SensorFINT Workshop "Smart Spectral Sensors for Agri-Food Quality and Process Control" that will be held in Porto (Portugal) from 16 to 17 September 2021: a must-attend event for the whole NDSS community.

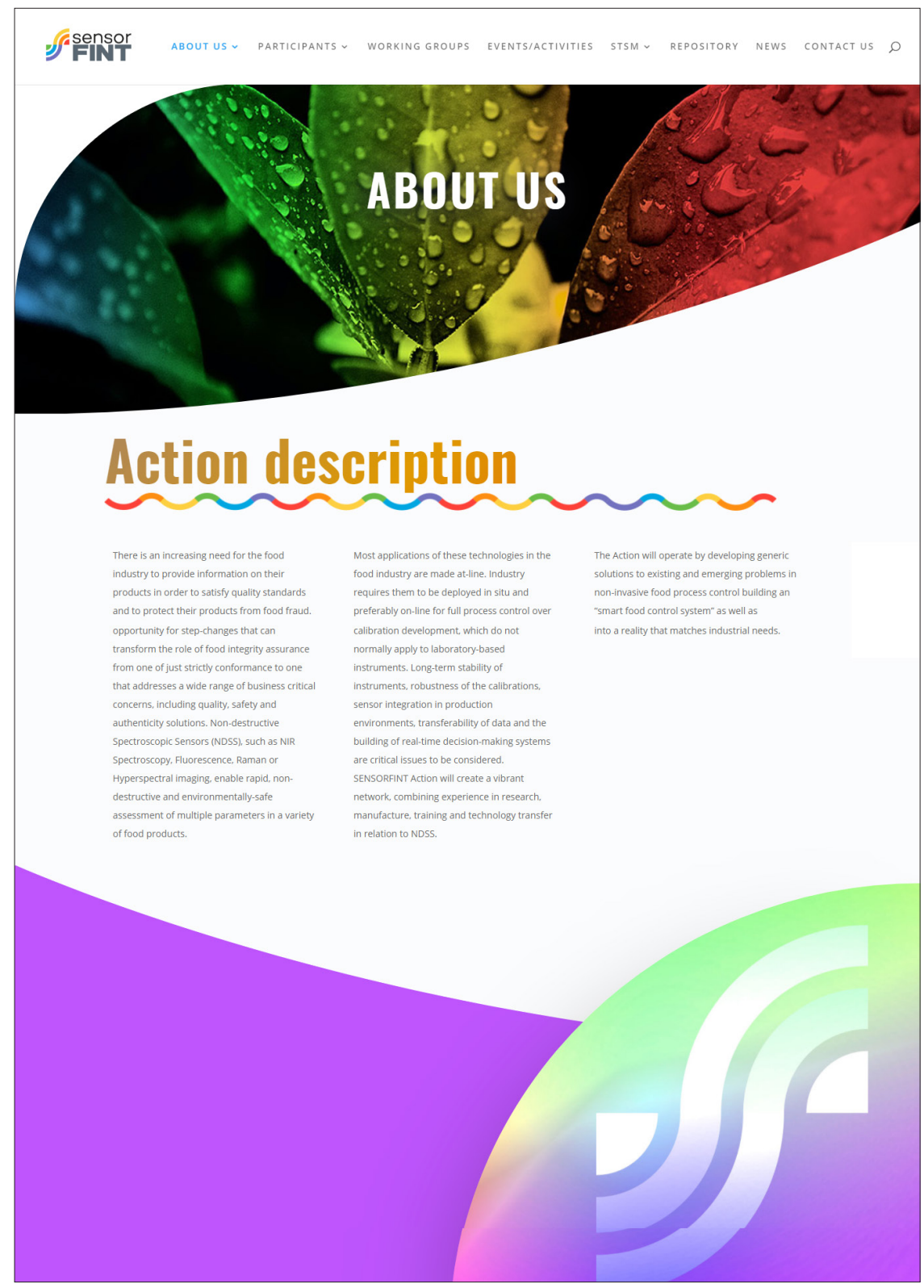

Figure 2. The SensorFINT website.

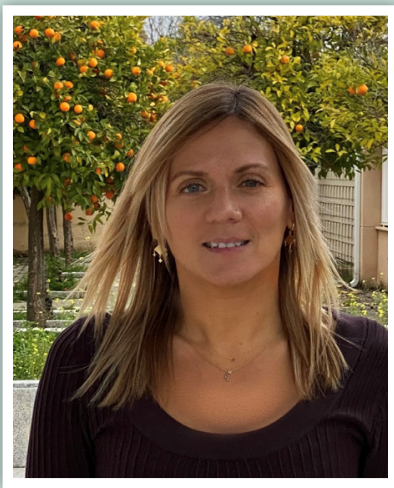

Dr Lola Peréz-Marín, Full-Professor in the Faculty of Agriculture and Forestry Engineering, University of Cordoba, Spain, is a worldwide-recognised expert in the use of NIR sensors for food integrity assessment. President of the Educational Committee of ICNIRS and Member of the International Scientific Advisory Board of the Institute for Global Food Security, Queen's University Belfast, UK. Recipient in 2014 of the international Tomas Hirschfeld Award and in 2020 of the international Gerald S. Birth Award for her outstanding contribution in the field of NIR spectroscopy. (iD) https://orcid.org/0000-0001-6629-4003 dcperez@uco.es 